\title{
IMAGES IN MEDICINE
}

\section{Papilloedema or pseudopapilloedema? Computed tomography to the rescue!}

A 61 year old man was investigated for suspected papilloedema (figl). He was completely asymptomatic and was not receiving any regular drug treatment. The finding was picked up on a routine visit to his optician. Incidentally, this was his first visit in 10 years. On examination, he was found to have no general or neurological signs apart from reduced colour vision and a relative afferent pupillary defect in the left eye. Baseline blood investigations were normal. Computed tomography of his head was normal apart from a hyperdense appearance within the left optic nerve head (fig 2). A suspicion of optic nerve head drusen was confirmed by ocular ultrasonography (fig 3). The drusen were detected bilaterally although they were more prominent in the left eye.

Optic disc drusen can mimic papilloedema and can cause optic nerve dysfunction (relative afferent pupillary defect, ${ }^{1}$ visual field $\operatorname{loss}^{2}$ ) including anterior ischaemic optic neuropathy. ${ }^{3}$ Ocular B-scan ultrasonography has been shown to be the most sensitive investigation to pick up these lesions. ${ }^{4}$ It is also inexpensive and readily available in the eye clinic. We recommend computed tomography of the brain and orbits in papilloedema (in preference to magnetic resonance imaging) as a primary neurological imaging technique for its ability to identify this differential diagnosis in addition to existing indications like identification of CNS mass lesions and being a pre-requisite to lumbar puncture. The examining physician and the reporting radiologist must entertain this diagnosis when dealing with atypical cases of disc swelling.

P T Ashwin, R R Sivaraj, L Wehbeh Department of Ophthalmology, West Midlands Deanery, West Midlands, UK

Correspondence to: Dr P T Ashwin, 3 Oakhill Drive, Brierley Hill, West Midlands DY5 3PP, UK; pammal.ashwin@gmail.com

Competing interests: none declared.

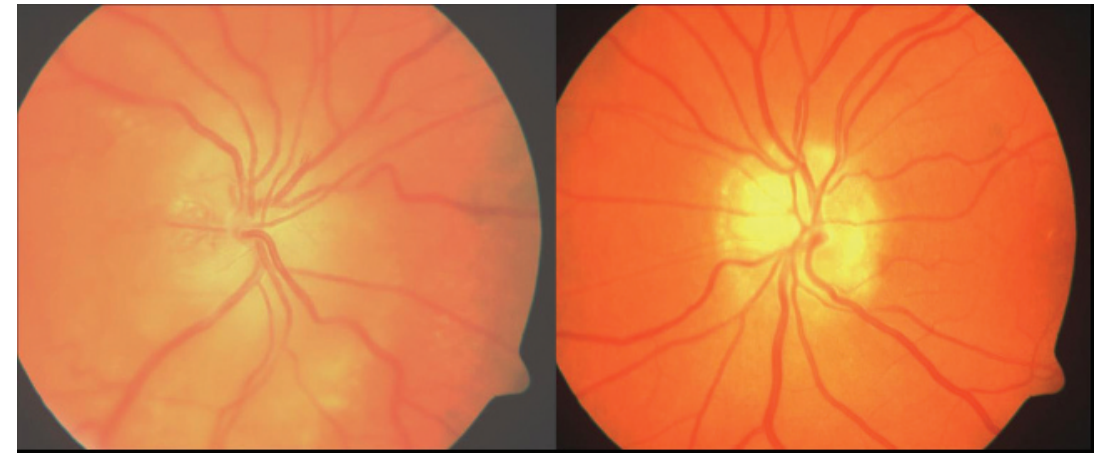

Figure 1 Colour fundus photograph showing bilateral optic disc swelling.

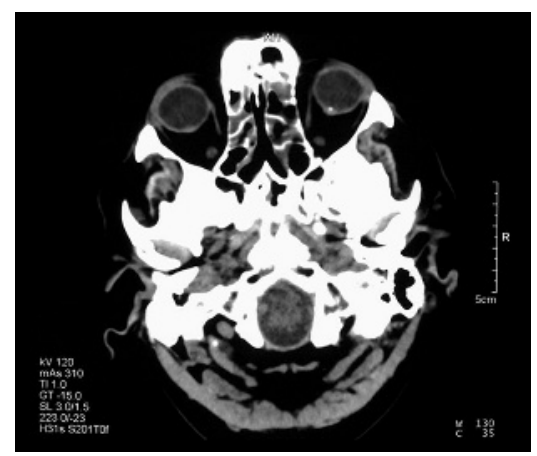

Figure 2 Axial computed tomography of the brain and orbits showing a hyperdense mass in the left optic nerve head.

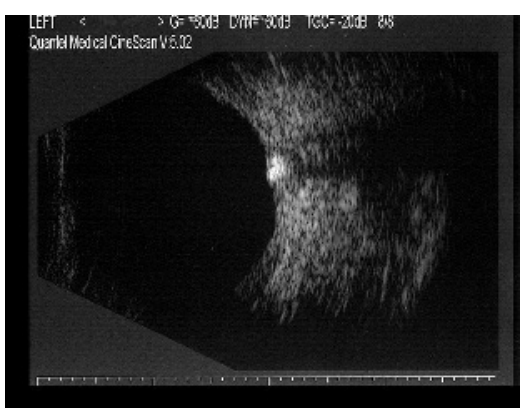

Figure 3 Ocular ultrasonogram showing optic disc drusen.

\section{REFERENCES}

1 Vicary DL, Swann PG. Relative afferent pupil defect associated with optic disc drusen. Aust N Z J Ophthalmol 1991;19:245-8.

2 Frangie JP, Nepomnyaschy N. Visual field loss and a relative afferent pupillary defect in a patient with optic nerve head drusen. J Ophthalmic Nurs Technol 1991;10:194-200.

3 Liew SC, Mitchell P. Anterior ischaemic optic neuropathy in a patient with optic disc drusen. Aust N Z J Ophthalmol 1999;27:157-60.

4 Kurz-Levin MM, Landau K. A comparison of imaging techniques for diagnosing drusen of the optic nerve head. Arch Ophthalmol 1999;117:1045-9. 